\title{
Relato de Caso de Doença Cardíaca Valvular Complicada com um Cisto Sanguíneo no Átrio Direito. Revisão da Literatura
}

\author{
A Case Report of Valvular Heart Disease Complicated with a Blood Cyst in Right Atrium. Review of the Literature
}

Shu Jiang, ${ }^{1 \oplus}$ Xiao-Cong Wang, ${ }^{1}{ }^{\oplus}$ Yan-Li Zhang, ${ }^{1}$ Wei Yu, ${ }^{1}$ Li-Ping Pei, ${ }^{1}$ Yan Ma ${ }^{1}$

Jilin University First Hospital, ${ }^{1}$ Changchun - China

\section{Introdução}

Os cistos sanguíneos no coração são frequentemente reconhecidos como benignos. Geralmente não causam sintomas clínicos e são frequentemente encontrados em bebês menores de dois meses. ${ }^{1} \mathrm{Na}$ maioria dos casos, os cistos sanguíneos estão nas válvulas cardíacas ou suas estruturas de suporte. ${ }^{2}$ Os cistos cardíacos em adultos são extremamente raros, especialmente nas câmaras do coração. Aqui, relatamos um caso de doença cardíaca valvular complicada com um cisto sanguíneo no átrio direito. As ecocardiografias transtorácica, de contraste e esofágica foram utilizadas para examinar o paciente.

\section{Relato de caso}

A paciente era uma mulher de 64 anos com palpitações e falta de ar após o exercício por quatro meses. Nas duas semanas anteriores, os sintomas haviam piorado. Os hospitais locais recomendaram encaminhamento para hospitais superiores, e assim foi encaminhada ao nosso hospital para tratamento. Ela tinha um histórico de hipertensão por sete anos, fumante durante 30 anos e cirurgia de histeromisma há mais de 20 anos. Não tinha histórico de diabetes e negava tuberculose, hepatite e outras doenças infecciosas. O paciente não tinha um registro claro de medicação. Na admissão, seus sinais vitais estavam estáveis. O eletrocardiograma mostrou fibrilação atrial. Murmúrios sistólicos puderam ser ouvidos na área de auscultação da válvula mitral. Não houve murmúrios em outras áreas de auscultação de válvulas.

A ecocardiografia transtorácica revelou átrios aumentados bilateralmente, estenose mitral leve e insuficiência grave, com função sistólica ventricular esquerda normal (FEVE $=52 \%$ ). Além disso, tinha uma lesão anecóica circular de aproximadamente $30 \times 41 \mathrm{~mm}$ de tamanho no átrio direito, que não causava obstrução da válvula tricúspide (Figura 1A).

Um dispositivo de diagnóstico de ultrassom GE Vivid E9 com uma sonda M5sC foi usado para uma ecocardiografia de

\section{Palavras-chave}

Cistos Sanguíneos/genética; Cistos /cirurgia; Doença das Valvas Cardíacas; Átrio do Coração/fisiologia; Função Atrial; Ecocardiografia/métodos.

\author{
Correspondência: Xiao-Cong Wang • \\ Jilin University First Hospital - The First Hospital of Jilin University, 1 Xin Min \\ Street, Changchun, Jilin Changchun 130021 - China \\ E-mail: 247266443@qq.com \\ Artigo recebido em 26/01/2021, revisado em 20/05/2021, aceito em \\ $16 / 06 / 2021$
}

DOI: https://doi.org/10.36660/abc.20210063 contraste examinação. SonoVue $(1 \mathrm{ml})$ foi injetado lentamente através da veia cubital mediana durante 1 minuto. $\mathrm{O}$ artefato de cintilação foi acionado aproximadamente 2 minutos após a injeção do agente de contraste. As microbolhas no miocárdio foram destruídas rapidamente quando um pulso de alta IM foi emitido. Em seguida, o processo de enchimento do agente de contraste no miocárdio e tumores foi observado em um estado de baixa IM. A imagem de perfusão contrastada mostrou estrutura cística com septo de aproximadamente 36×42 mm no átrio direito e ausência de perfusão na massa. (Figura 1B, C).

Ecocardiografia transesofágica intraoperatória revelou eco circular em átrio direito, com contraste ecocardiográfico espontâneo e septação, o que consideramos cisto sanguíneo (Figure 1D).

O paciente foi submetido à excisão de massa atrial direita, substituição da válvula mitral e valvuloplastia tricúspide. A massa estava localizada no átrio direito ligado à fossa oval, perto do orifício da veia cava inferior, por um pedículo de aproximadamente $5 \mathrm{~mm}$ de diâmetro. Tinha cerca de $40 \mathrm{x}$ 40 mm de tamanho (Figura 2A, B). A cápsula estava intacta, dura e roxa-preta. $\mathrm{O}$ tecido septo atrial conectado ao tumor e ao pedículo foi completamente removido. Foi realizada substituição da válvula mitral e valvuloplastia tricúspide.

O exame macroscópico mostrou que a massa do átrio direito era redonda com um volume de $45 \times 35 \times 27 \mathrm{~mm}$, tinha uma cápsula completa e um pedículo estreito na superfície. A seção de massa era unilocular, a parede cística tinha 1-2 mm de espessura, o revestimento interno era liso, e o conteúdo eram principalmente coagulos. O exame microscópico de luz de seções embebidas em parafina mostrou tecido semelhante a parede fibrocística, hiperplasia de tecido fibroso intersticial, degeneração hialina, degeneração mucinosa na massa do átrio direito e tecido de coagulação maciço, o que estava de acordo com as alterações do cisto (Figura 3A, B). O tecido da válvula examinado mostrou degeneração de hialina e degeneração mucinosa com infiltração dispersa de células inflamatórias crônicas (Figura 3C).

O paciente se recuperou bem após a operação. Nenhuma massa anormal foi encontrada no átrio direito por ecocardiografia. Além disso, sua prótese de válvula mitral e a função da válvula tricúspide estavam normais.

\section{Discussão}

Elsasser relatou pela primeira vez cistos sanguíneos em 1844. ${ }^{3}$ Geralmente são de origem congênita e são encontrados poucos meses após o nascimento, desaparecendo espontaneamente com o tempo. Eles ocorrem principalmente em válvulas cardíacas, como válvulas mitral, tricúspide, 


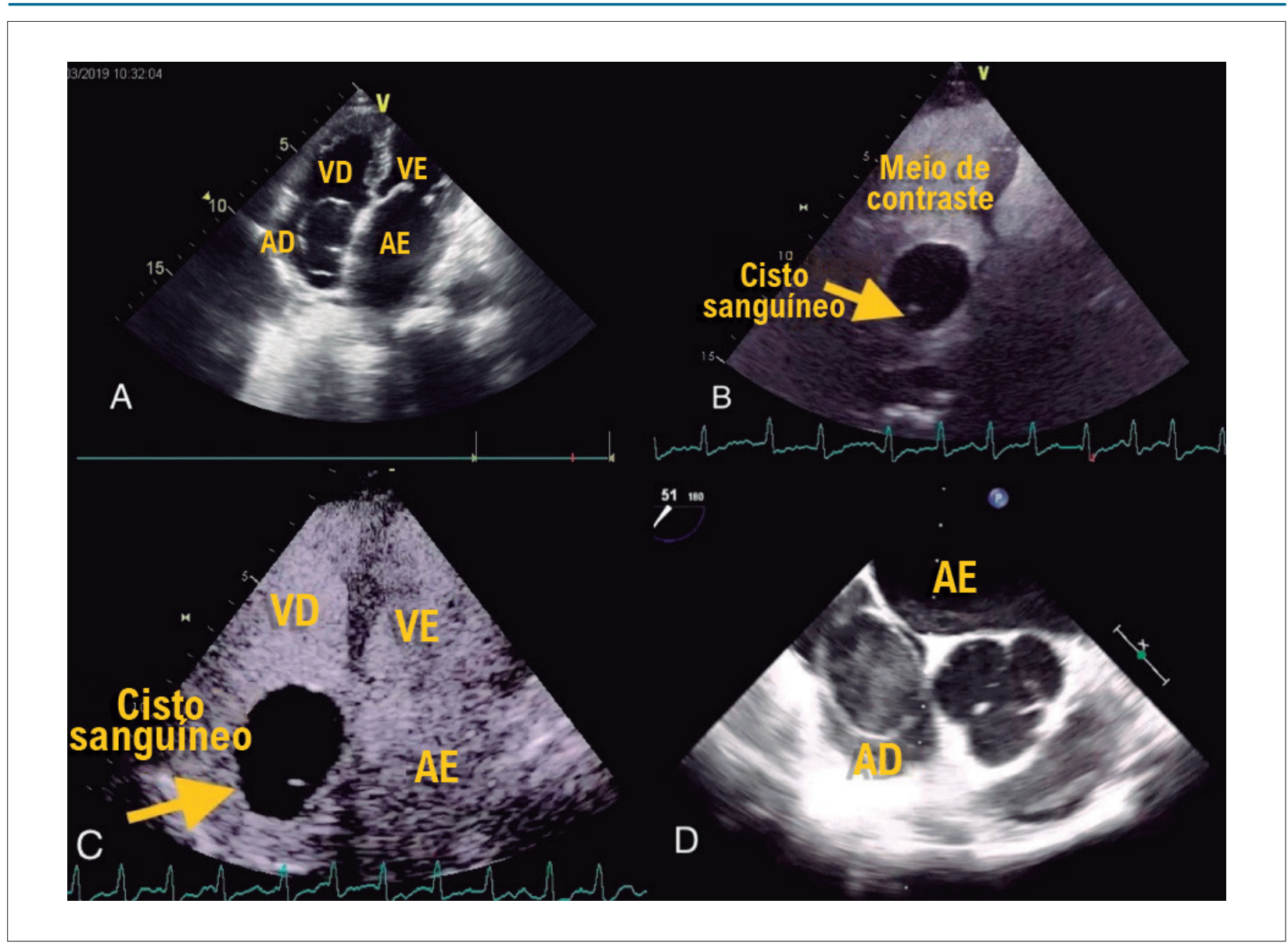

Figura 1 - A) A ecocardiografia transtorácica mostrou lesão anecóica circular no átrio direito. $B, C) A$ imagem de perfusão de contraste mostrou uma estrutura cística com septo e ausência de perfusão na massa. D) A ecocardiografia transesofágica intraoperatória revelou um eco circular no átrio direito, com contraste ecocardiográfico espontâneo e septação. VD: ventrículo direito; VE: ventrículo esquerdo; AD: átrio direito; $A E$ : átrio esquerdo.

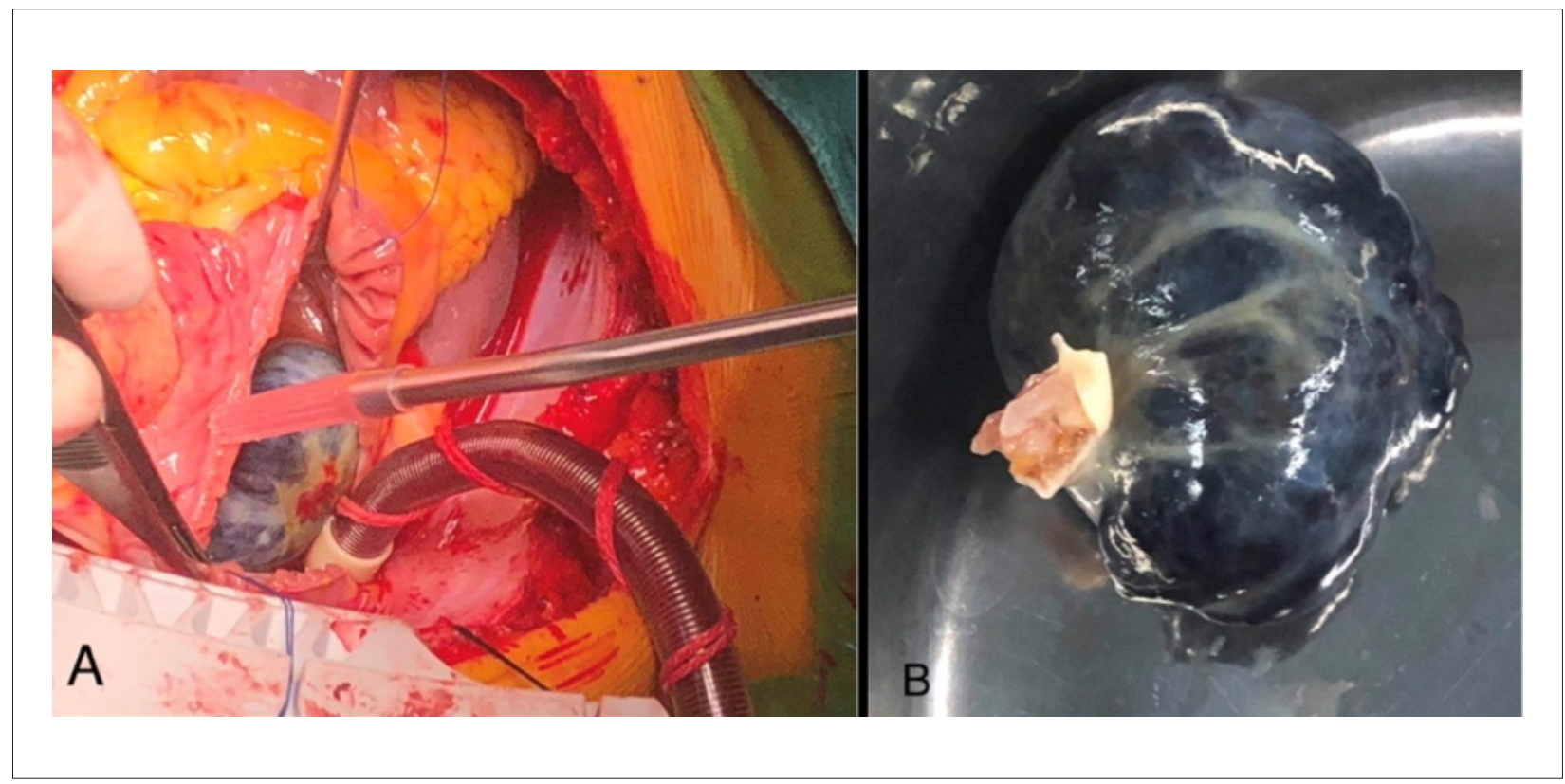

Figura $2-A, B)$ Achados cirúrgicos. 0 tumor estava localizado no átrio direito ligado à fossa oval, perto do orificio da veia cava inferior, por um pedículo de aproximadamente $5 \mathrm{~mm}$ de diâmetro. 


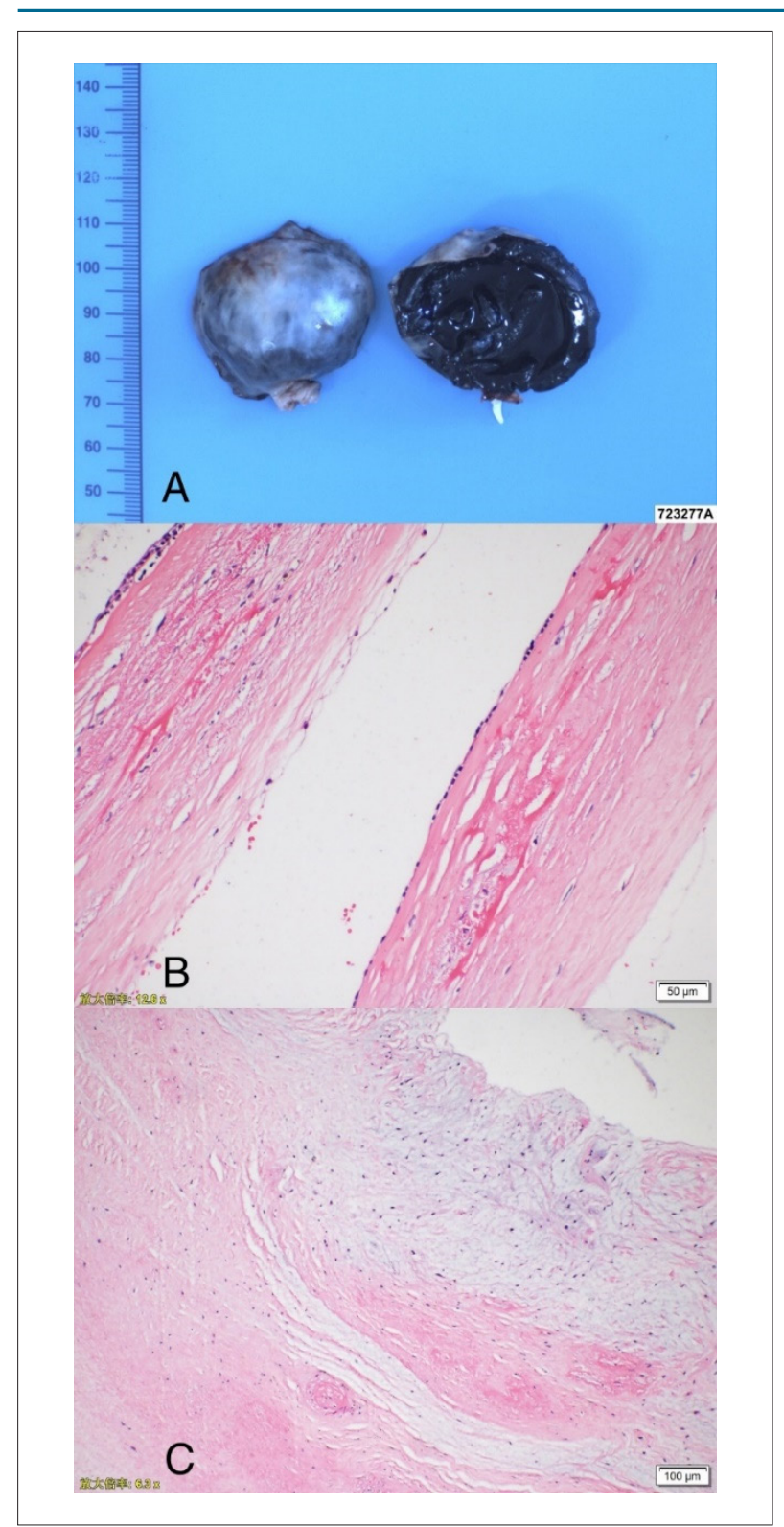

Figura $3-A, B)$ Achados patológicos. A massa foi preenchida com tecido fibrocístico semelhante à parede, hiperplasia de tecido fibroso intersticial, degeneração de hialina e degeneração mucinosa na massa do átrio direito, e tecido de coagulação maciça. C: 0 tecido da válvula examinado mostrou degeneração de hialina e degeneração mucinosa com infiltração dispersa de células inflamatórias crônicas.

aórtica e válvulas pulmonares. Às vezes, eles existem no átrio esquerdo e átrio direito. ${ }^{4,5}$ Cistos sanguíneos em adultos são extremamente raros, especialmente na cavidade cardíaca. Existem várias hipóteses sobre as causas da formação do hemocisto: 1. Durante o desenvolvimento das válvulas, cistos de sangue são formados porque o sangue é espremido e fica preso na lacuna que posteriormente se fecha. 2. Possíveis alterações heteroplásicas no tecido mesotelial pericárdico primário. 3. Sakakibara et al., ${ }^{6}$ sugerem que um bloqueio repentino da circulação causa cistos sanguíneos no átrio ou ventrículo. Eles acreditavam que as tendências de hipóxia, inflamação e sangramento poderiam ser responsáveis pela transformação do hematoma endocárdico em hemocistos. 4. Os cistos sanguíneos representam apenas vasos sanguíneos dilatados. 5. Degeneração mucinosa. ${ }^{7}$ No entanto, ainda não há consenso sobre a causa dos cistos sanguíneos. ${ }^{4,8,9} \mathrm{~A}$ paciente atual tinha insuficiência mitral. O exame patológico da válvula mostrou degeneração de hialina e degeneração mucinosaa. Presume-se que isso tenha relação com a formação de cistos sanguíneos.

Os tumores cardíacos são principalmente benignos e mixomatosos. Eles podem ocorrer em qualquer idade. O mixoma geralmente ocorre na superfície do endocárdio da cavidade cardíaca, 60\% -80\% das vezes no átrio esquerdo. O pedículo se prende à fossa oval do septo atrial e tem uma certa amplitude de movimento com o ciclo cardíaco.

No entanto, o mixoma é geralmente uma massa de eco forte redondo ou oval com ecos internos regulares. Se houver necrose no centro, pode ser uma área anecóica.

A ecocardiografia de contraste do ventrículo esquerdo de mixoma mostra que o meio de contraste é esparso, mas realçado na lesão. Sua intensidade é inferior à do tecido miocárdico adjacente, o que não condiz com o caso.

As manifestações ecocardiográficas da equinococose cardíaca são lesões císticas que ocupam espaço no coração. A distribuição de cistos no coração está relacionada principalmente com o suprimento de sangue do miocárdio. Portanto, o local mais comum é o miocárdio da parede ventricular, que tem o maior suprimento de sangue do coração, seguido pela parede atrial, e a cavidade cardíaca é menos frequentemente envolvida. ${ }^{10}$ No entanto, o paciente não tinha uma história clara de contato com bovinos ou ovinos ou uma dieta suja, de modo que o diagnóstico não foi apoiado.

De 1958 a 2020, havia apenas dez artigos sobre cistos sanguíneos no átrio direito confirmados por patologia. Nossa revisão da literatura em língua inglesa revelou dez outras entradas desde 1996, descrevendo um total de 10 pacientes com detalhes desses cistos sanguíneos no átrio direito (Tabela 1). Os sintomas dos cistos sanguíneos relatados variam. Quatro pacientes eram assintomáticos. ${ }^{11-14}$ Outros pacientes apresentaram sintomas. Os sintomas comuns foram dispneia e desconforto no peito, um caso de dor crônica e um de dor de cabeça. O local de apresentação também foi diferente. Seis cistos foram no septo atrial, ${ }^{11,13-17} 2$ na fossa oval, ${ }^{12,18} 1$ na válvula tricúspide ${ }^{19}$ e 1 na válvula sinusal coronária. ${ }^{20} \mathrm{~A}$ calcificação foi encontrada em 5 dos cistos sanguíneos. ${ }^{12,14-16,18}$ Após a cirurgia, a maioria dos pacientes se recuperou1, 11-15,18,20 2 pacientes não tiveram um prognóstico explicado ${ }^{16,19}$ e 1 paciente morreu. ${ }^{17}$

Atualmente, não existe um padrão uniforme para o tratamento de cistos sanguíneos. Portanto, os pacientes com sintomas podem ser acompanhados regularmente. Relatase que os hemocistos podem causar obstrução do trato de saída ventricular esquerda, disfunção da válvula, disfunção ventricular, derrame embólico, embolia pulmonar e obstrução da artéria coronária. ${ }^{8}$ Portanto, para pacientes com sintomas, deve ser realizado tratamento cirúrgico precoce. 
Carta Científica

Tabela 1 - Resumo clínico dos casos de cistos sanguíneos encontrados na literatura inglesa desde 1958

\begin{tabular}{|c|c|c|c|c|c|c|c|c|c|c|}
\hline Paciente & $\begin{array}{l}\text { Primeiro } \\
\text { autor }\end{array}$ & Ano & Gênero & Idade & $\begin{array}{c}\text { Tamanho do } \\
\text { tumor }\end{array}$ & Local & $\begin{array}{c}\text { Cisto } \\
\text { sanguíneo } \\
\text { com cálcio }\end{array}$ & Sintoma & Histórico & Prognóstico \\
\hline 1 & H Niinam ${ }^{15}$ & 1996 & $\mathrm{~F}$ & 59 & $20 \times 20 \mathrm{~mm}$ & $\begin{array}{l}\text { septo atrial } \\
\text { entre } 0 \\
\text { fossa ovalis } \\
\text { e tricúsulide } \\
\quad \text { válvula }\end{array}$ & Sim & $\begin{array}{c}\text { episódios } \\
\text { periódicos } \\
\text { de pressão } \\
\text { substernal e } \\
\text { tosse seca }\end{array}$ & nenhum & Recuperação \\
\hline 2 & $\begin{array}{l}\text { Hiroyuki } \\
\text { Tanaka }^{11}\end{array}$ & 2003 & M & 52 & $40 \times 30 \mathrm{~mm}$ & $\begin{array}{l}\text { septo atrial } \\
\text { entre o } \\
\text { fossa ovalis } \\
\text { e válvula } \\
\text { tricúspide }\end{array}$ & Não & nenhum & câncer gástrico & Recuperação \\
\hline 3 & $\begin{array}{c}\text { Gernot } \\
\text { Seebacher }^{18}\end{array}$ & 2006 & $\mathrm{~F}$ & 65 & $44 \times 20 \mathrm{~mm}$ & $\begin{array}{c}\text { fossa ovalis } \\
\text { de septo } \\
\text { atrial }\end{array}$ & Sim & $\begin{array}{l}\text { dispneia, } \\
\text { angina e } \\
\text { taquicardia }\end{array}$ & nenhum & Recuperação \\
\hline 4 & $\begin{array}{c}\text { Kaoru } \\
\text { Otsuka }^{12}\end{array}$ & 2007 & M & 56 & $30 \times 20 \mathrm{~mm}$ & $\begin{array}{c}\text { fossa ovalis } \\
\text { do septo } \\
\text { atrial }\end{array}$ & Sim & nenhum & nenhum & Recuperação \\
\hline 5 & $\begin{array}{l}\text { Tomasa } \\
\text { Centella }^{16}\end{array}$ & 2015 & Desconhecido & 62 & $28 \times 28 \mathrm{~mm}$ & septo atrial & Sim & $\begin{array}{l}\text { dor de } \\
\text { cabeça }\end{array}$ & $\begin{array}{l}\text { ferritina elevada } \\
\text { não relacionada } \\
\text { ao gene de } \\
\text { hemocromatose, } \\
\text { uma hérnia de } \\
\text { hiato, esofagite } \\
\text { leve e cistos de } \\
\text { seio renal }\end{array}$ & Desconhecido \\
\hline 6 & $\begin{array}{l}\text { Hiroyuki } \\
\text { Otsuka }^{13}\end{array}$ & 2016 & $\mathrm{~F}$ & 85 & $\begin{array}{l}30 \times 30 \mathrm{~mm}, \\
25 \times 25 \mathrm{~mm}\end{array}$ & $\begin{array}{l}\text { septo atrial } \\
\text { entre o } \\
\text { fossa ovalis } \\
\text { ea válvula } \\
\text { tricúspide }\end{array}$ & Não & nenhum & $\begin{array}{l}\text { síndrome do } \\
\text { nódulo sinusal }\end{array}$ & Recuperação \\
\hline 7 & $\begin{array}{l}\text { Hilary } \\
\text { Bews }^{19}\end{array}$ & 2018 & $\mathrm{~F}$ & 62 & $45 \times 54 \mathrm{~mm}$ & $\begin{array}{l}\text { válvula } \\
\text { tricúspide }\end{array}$ & Não & $\begin{array}{l}\text { dor crônica } \\
\text { nas costas }\end{array}$ & nenhum & Desconhecido \\
\hline 8 & $\begin{array}{l}\text { Feridoun } \\
\text { Sabzi }^{20}\end{array}$ & 2019 & M & 76 & Desconhecido & $\begin{array}{c}\text { válvula } \\
\text { sinuso } \\
\text { coronariana }\end{array}$ & Desconhecido & dispneia & nenhum & Recuperação \\
\hline 9 & $\begin{array}{c}\text { Behnam } \\
\text { Shakerian }^{14}\end{array}$ & 2019 & $M$ & 73 & $16 \times 10 \mathrm{~mm}$ & septo atrial & Sim & nenhum & nenhum & Recuperação \\
\hline 10 & A Angelov ${ }^{17}$ & 2012 & $\mathrm{~F}$ & 28 & $60 \times 65 \mathrm{~mm}$ & septo atrial & Não & $\begin{array}{l}\text { desconforto } \\
\text { no peito, } \\
\text { falta de ar e } \\
\text { febre }\end{array}$ & nenhum & Morte \\
\hline
\end{tabular}

\section{Conclusão}

A ecocardiografia tornou-se a primeira escolha para diagnosticar cistos sanguíneos porque pode nos permitir observar o tamanho, forma, estrutura, função e desenvolvimento valvular do coração, e é não invasiva e segura. Além disso, a ecocardiografia com contraste é útil no diagnóstico de cistos sanguíneos porque pode mostrar se há um preenchimento com meio de contraste na massa. Além disso, a ecocardiografia transesofágica intraoperatória pode ser usada para localizar a massa cardíaca e, assim, ajudar na operação.

\section{Agradecimentos}

Os autores gostariam de agradecer à paciente e sua família por permitirem o uso e publicação de seu prontuário neste relato de caso.

\section{Contribuição dos autores}

Concepção e desenho da pesquisa e Redação do manuscrito: Jiang S; Obtenção de dados: Yu W; Análise e interpretação dos dados: Li-Ping P, Yan M; Revisão crítica do manuscrito quanto ao conteúdo intelectual importante: Xiao-Cong W, Yan-Li Z. 


\section{Carta Científica}

\section{Potencial conflito de interesse}

Não há conflito com o presente artigo

\section{Fontes de financiamento}

O presente estudo não teve fontes de financiamento externas.

\section{Referências}

1. Sharma S, Strauss R, Swanson JS. Giant blood cyst of the mitral valve. Asian Cardiovasc Thorac Ann; 2000;8(3):278-9.

2. Kuhn A, Schreiber C, Weirich G, Vogt M.Intracardiacbloodcyst: rare finding in a complex congenital heart lesion. Eur Heart J. 2012;33(2):229.

3. Elsasser C. Bericht uber die Ereignisse in der gebaranstalt des Catherinen Hospital im Jahre 1844. Med Correspondenzblatt.1844;14(2):297.

4. Dencker M, Jexmark T, Hansen F, Tyden PC, Roijer A, Luhrs C. Bileaflet blood cysts on the mitral valve in an adult. J Am Soc Echocardiogr. 2009;22(9): 1085.e5-1085.e8

5. Zimmerman KG, Paplanus SH, Dong S, Nagle RB. Congenital blood cysts of heart valves. Human Pathol. 1983;14(8):699-703.

6. Sakakibara S, Katsuhara K, lida Y, Nishida H. Pulmonary subvalvular tumor. Dis Chest 1967;51(6):637-42.

7. Ozmen G, Demir M, Arı H, Aktas I. A rare association: concomitant presence of mitral valve blood cyst with atrial septal aneurysm and cor triatriatum dexter. Anatol J Cardiol. 2015; 15(6):502-3.

8. Minneci C, Casolo G, Popoff G, Sulla A, Comin CE, Pedemonti E. A rare case of left ventricular outflow obstruction. Eur J Echocardiogr. $2004 ; 5(1): 72-5$

9. Xie SW, Lu OL, Picard MH. Blood cyst of the mitral valve: detection by transthoracic and transesophageal echocardiography. J Am Soc Echocardiogr 1992;5(5):547-50.

10. Djoshibaev S, Kudaiberdiev T, Maralov A, Shabralier S, Djooshiv K, Halikov UM, et al. Surgical treatment of isolated cardiac echinococciasis: report of five cases. Anadolu Kardiyol Derg 2003;3(2):137-43.

\section{Vinculação acadêmica}

Não há vinculação deste estudo a programas de pós-graduação.

\section{Aprovação ética e consentimento informado}

Este artigo não contém estudos com humanos ou animais realizados por nenhum dos autores.

11. Hiroyuki T, Mio E, Takashi N, Narisawa T, Mori T, Masuda M, etal. Atrial Blood Cyst With Ischemic Heart Disease. Circ J. 2003;67(1): 91-2.

12. Ptsuka K, Tonari LA, Limori A, Shimomura H, Ko T, Kitaura Y, et al. Right atrial blood cyst with total occlusion of the right coronary artery. Heart Vessels .2007;22(3):208-10.

13. Hiroyuki O, Kouichi A, Toshifumi F,Takasya T, Shojima T, Takagi K, etal. Double Right Atrial Blood Cysts. Ann Thorac Surg.2016;101(5):147-9 .

14. Behnam S, Mohammad J. Right Atrium Blood Cyst and Calcified Kernel in an Adult. Clin Med Insights Case Rep. 2019 Sep 29;12:117954-6198740807

15. H Niinami, S Ishihara, S Hara Tanaka S,Yamaguti E,Takase S. Blood cyst with a calcium stone originating from the right atrial septum. Cardiovasc Surg. 1996;4(2):260-2.

16. Centella T, Moya JL, Munoz M, Reguero EM. Giant Endocardial Blood Cyst in the Right Atrium Echocardiographic and Magnetic Resonance Imaging Features. Circulation. 2008;117(12):3250-1.

17. A Angelov, Y Yotov, K Kalchev,et al. Right atrium giant blood cyst in a young female with acute pericarditis, complicated with fatal cardiac tamponade. Int J Cardiol. 2013;163(2):31-32.

18. Seebacher G, Binder T, Frank H, Wolner E, Mohl W. Cystic Formation of the Foramen Ovale Mimicking a Right Atrial Myxoma.Ann Thorac Surg .2006;82(6)::2296-8.

19. Bewstt H, Strzelzyk J, Luqman Z, Jassal D. Multimodality imaging of a right atrial blood cyst. Eur Heart J. 2017;38(48):3603.

20. Sabzi F, Heydari A, Asadmobini A, Heidari MB. Right Atrial Blood Cyst with Stones Suspended from the Coronary Sinus. Sultan Qaboos Univ Med J. $2019,9(2): 161-3$ 